\title{
Sensitivity and Sensing Depth Analysis of Open-ended Contact Probes for Cancer Diagnosis
}

\author{
Cemanur Aydinalp, Sulayman Joof, Ibrahim Akduman, and Tuba Yilmaz \\ Department of Electronics and Communication Engineering, Istanbul Technical University, Istanbul \\ Technical University, Maslak, Istanbul 34469, Turkey
}

\begin{abstract}
Open-ended contact probes have been widely utilized in laboratory environment to quantify the dielectric properties of high permittivity and lossy materials such as biological tissues and other lossy liquids. At microwave frequencies, the dielectric properties of biological tissues have been quantified with open-ended contact probes method with the motivation of building microwave diagnostic and therapeutic technologies. The method is preferred to other microwave dielectric property measurement techniques due to broadband measurement capabilities and minimal sample preparation requirements. However, the measurement procedure is cumbersome and the measurements tend to be error-prone. The commercial probes can have error rates as high as $5 \%$ and it can increase with the cable movements, calibration degradation, and probe wear-off. Although the technique is powerful and able to measure the inherent dielectric property discrepancy between different tissues the applications are confined to laboratory use. To explore the true potential of this technique and enable the transition of this technique to a diagnostic technology, there is a need to improve the technique and analyze the performance in a layered heterogeneous medium. In this work, we performed simulations and measurements with open-ended contact probes having different aperture diameters. The probes are built with copper and Teflon sandwiched between inner and outer conductors. Measurements with openended coaxial probes are performed on layered medium emulating the dielectric properties of the biological tissues to analyze the sensing depth and sensitivity of four in-house fabricated openended coaxial probes. An in-house algorithm is utilized to retrieve the dielectric properties of the mediums utilized during this study.
\end{abstract}

\section{INTRODUCTION}

Dielectric properties of biological tissues at microwave frequencies have long been of interest to researchers for developing medical diagnostic, monitoring, and therapeutic technologies such as microwave imaging and ablation $[1,2,3]$. Furthermore, these properties are of utmost importance not only for the early design stage of medical devices, but also for test stage. Therefore, the accurate knowledge of dielectric properties is critical for development of new medical devices. Thus, many researchers have focused on expanding the dielectric property database of biological tissues in the past decade. In order to measure the dielectric properties of biological tissues, among other methods open-ended coaxial probe has been employed due to the advantages the technique including the broadband measurement capability and flexible sample size requirements. The minimal sample preparation requirement of the technique enables the non-destructive measurement capability which led the researchers to prefer this method for in-vivo measurement of biological tissues [4, 5]. Even though utilizing open-ended coaxial probes has a number of advantages, the technique suffers from equipment-related, tissue-related and other measurement errors [6]. Equipment-related errors can stem from the probe, cable, connectors, and drift introduced by the S-parameter measurement unit [6]. Tissue-related errors result from the change in temperature of the sample, probe-sample contact, probe-sample pressure, in vivo versus ex vivo experiments, sample handling procedure, tissue sample properties, and heterogeneity [6]. Additional errors can be introduced by the enduser an example is error stemming from the calibration. Since obtaining accurate dielectric property measurement is crucial, effect of aforementioned error sources should be minimized.For example, the probe-sample contact and the heterogeneity of target tissue can be controlled by using appropriate probe types. To overcome this challenge, the contact can b ensured by using flange-free probes and for heterogeneous tissues only targeted tissue can be included inside the probe sensing volume via utilization of probed with small apertures $[6,7]$.

Sensing depth have been analysed in the literature. In [8], a $2.2 \mathrm{~mm}$ diameter slim form probe from Agilent Technologies (Santa Clara, CA) was utilized to examine dielectric properties of twolayered structure composed of background liquid and a planar piece of Teflon. The dielectric 
properties measurement has been performed over the frequency range of $100 \mathrm{MHz} 8.5 \mathrm{GHz}$ with $100 \mathrm{MHz}$ intervals. The experiment has been operated by locating Teflon cylinder $0.0,0.175,0.325$ and $2.0 \mathrm{~mm}$ from the probe surface. During the measurements probe was in direct contact with Teflon, the measured dielectric properties equaled to pure Teflon properties. When the separation between the probe tip and Teflon cylinder was about $0.2 \mathrm{~mm}$, the dielectric property measurement showed approximately $50 \%$ dielectric properties of the intervening fluid. After the separation increased to $0.5 \mathrm{~mm}$, the measured properties reflected almost $90 \%$ of the liquid. The result of [8] presents that the most effective sensing volume for dielectric measurement with $2.2 \mathrm{~mm}$ diameter slim form probe is the first 200-400 microns of distance from the probe tip. In [9], the penetration depth has been investigated by performing experiment with probes named S15 and M25 two Delfin Technologies probes of diameters $18 \mathrm{~mm}$ and $21 \mathrm{~mm}$ respectively. The measurement system was composed of water tank and acrylic cylinder, and the dielectric properties have been measured at $300 \mathrm{MHz}$. In this study, the penetration depth has been defined as a margin where the permittivity values are equal to almost $75 \%$ of actual water properties instead of acrylic cylinder properties. The measured penetration depth for S15 and M25 were $1.84 \mathrm{~mm}$ and $2.74 \mathrm{~mm}$, respectively. In [10], the minimum breast tissue sample size that would have to be surgically resected to obtain the correct dielectric properties for a given probe size has been examined. For this reason, $2.2 \mathrm{~mm}$ and $3.58 \mathrm{~mm}$ diameter flange-free coaxial probe have been utilized to measure dielectric properties of liquid tissue simulants, which are ethanol, methanol and water, in the frequency range 1 to 20 GHz. In order to analyze sensing volume, the measurements have been performed at distances away from the side of the beaker in the radial direction range of 0.0 to $10.0 \mathrm{~mm}$ with $1.0 \mathrm{~mm}$ increments. The sensing depths for $2.2 \mathrm{~mm}$ diameter probe have been determined $0.75-1.0 \mathrm{~mm}$, 1.0-1.5 $\mathrm{mm}$ and $1.5 \mathrm{~mm}$ for ethanol, methanol, water, respectively. For $3.58 \mathrm{~mm}$ diameter probe has $1.25-1.5 \mathrm{~mm}, 2.25 \mathrm{~mm}, 2.5-3.0 \mathrm{~mm}$ sensing depths for ethanol, methanol, water, respectively. In [11], it has been aimed to minimize uncertainties in the dielectric measurement due to the longitudinal heterogeneities by analyzing correspondence between size of tissue in a specimen and effect of that tissue to dielectric properties measurement. In the experiment, $2.2 \mathrm{~mm}$ diameter slim form probe from Keysight Technologies (Santa Rosa, CA) has been utilized to measure dielectric properties of samples, which were deionized water, physiological $(0.9 \%)$ saline, acrylic, a rubberbased tissue-mimicking phantom, porcine muscle, porcine fat, and duck fat. The measurements have been performed for the sample in different thicknesses from $0.0 \mathrm{~mm}$ to $13.93 \mathrm{~mm}$ over the frequency range $300 \mathrm{MHz}$ to $8.5 \mathrm{GHz}$. For $2.2 \mathrm{~mm}$ slim form probe, histology depth has been investigated through five different scenarios at two different frequency point. Only two scenarios were constituted fully biological tissues which were duck fat-porcine muscle and duck fat-porcine fat. The results of these scenarios have showed histology depth are for duck fat-porcine muscle $2.776 \mathrm{~mm}$ and $2.750 \mathrm{~mm}$ at $300 \mathrm{MHz}$ and $8.5 \mathrm{GHz}$ respectively. Moreover, for duck fat-porcine fat histology depth $3.646 \mathrm{~mm}$ and $3.588 \mathrm{~mm}$ at $300 \mathrm{MHz}$ and $8.5 \mathrm{GHz}$ respectively.

In this work, we aim to analyze how sensing depth has been effected from probes dimension during the dielectric properties measurement via open-ended coaxial probe method. For this purpose, three probes have been designed with different aperture sizes, and the thickness of upper layer has been altered to analyze dielectric properties of two layered structure.

\section{METHOD}

CST Microwave Studio software (CST, Framingham, MA, USA) has been utilized to design the slim form probes with $2.2 \mathrm{~mm}, 0.9 \mathrm{~mm}$ and $0.5 \mathrm{~mm}$ aperture diameters given in Table 1 , and the length of probes have been determined at $200 \mathrm{~mm}$. The impedance of probes has been matched at $50 \mathrm{Ohm}$. Furthermore, each probe has been simulated with two-layered structure which were composed of upper layer skin tissue and a fixed Perfect Electric Conductor (PEC) 40mm lower layer. The thickness of the upper layer is varied mainly within the range of 0.1 and $2 \mathrm{~mm}$ with small increments to evaluate the sensing depths of the probes. Furthermore, the two-layered structure has been designed as a square with $17 \mathrm{~mm}$ edges. The simulations have been performed over the frequency range of $500 \mathrm{MHz}$ to $2 \mathrm{GHz}$. The simulated probes have been calibrated with the standard three-load calibration procedure: open circuit, short circuit, and a broadband load that is a liquid with known dielectric properties generally distilled water is used in practice. In addition, since the aperture sizes for $0.9 \mathrm{~mm}$ and $0.5 \mathrm{~mm}$ are small, in order to have a better simulation results, the mesh size properties. The simulation configuration is given in Fig. 1.

The obtained S-parameters from calibration and two layered structure have been applied inhouse algorithm to compute the dielectric properties. First order Debye parameters for skin tissue 

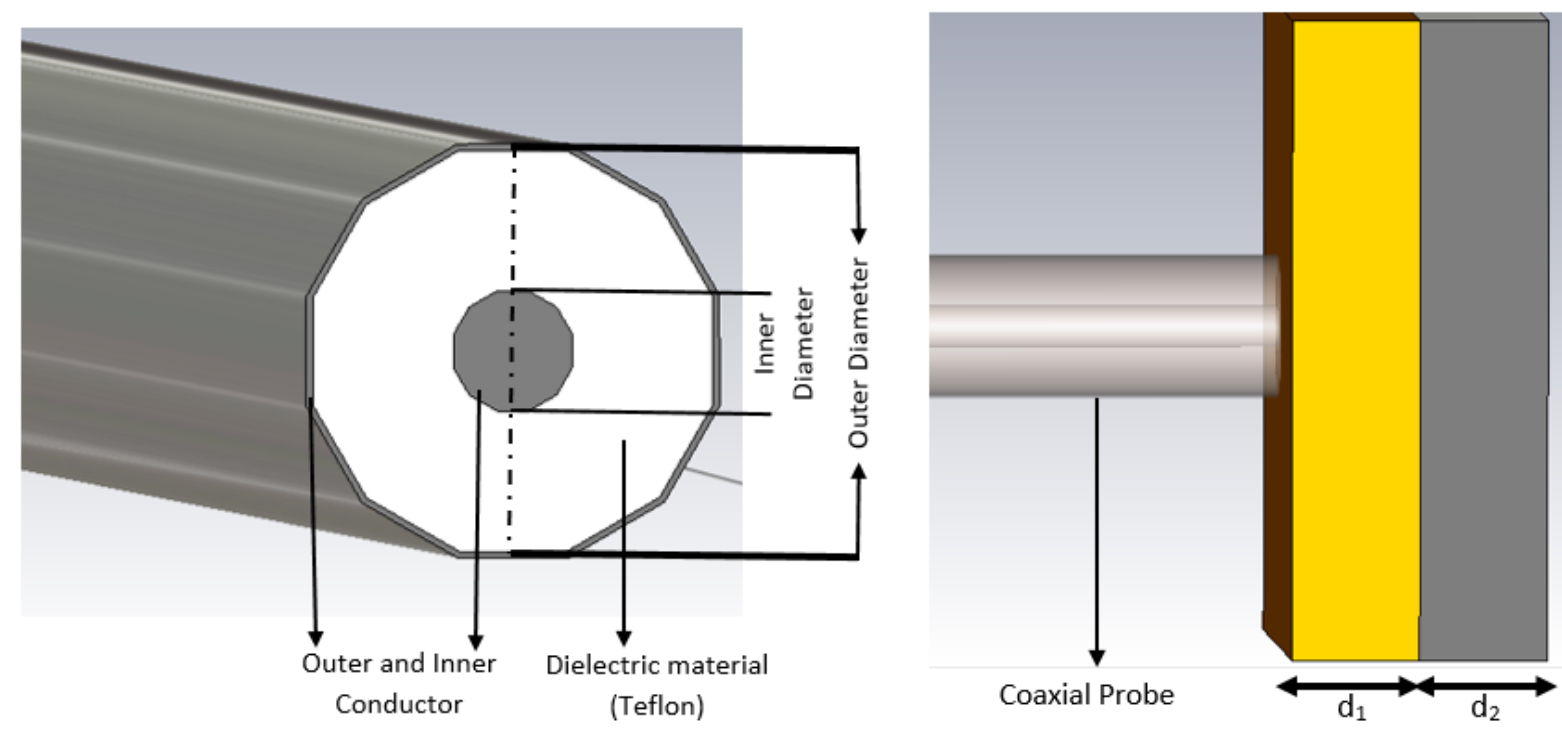

Figure 1: Coaxial Probe Geometry with two-layered structure from (a) topview, (b) sideview.

Table 1: Simulated three $50 \Omega$ probes dimensions

\begin{tabular}{cccc}
\hline Inner Diameter $(\mathrm{mm})$ & Outer Diameter $(\mathrm{mm})$ & Dielectric Type & $\epsilon_{r}$ \\
\hline 0.5 & 0.15 & $P T F E$ & 2.1 \\
0.9 & 0.28 & $P T F E$ & 2.1 \\
2.2 & 0.65 & $P T F E$ & 2.1 \\
\hline
\end{tabular}

Table 2: Debye parameters of skin tissue for two-layered structure

\begin{tabular}{cccc} 
Material & $\epsilon_{r}$ & $\sigma(S / m)$ & $\tau(p s)$ \\
Skin Tissue & 30.75 & 0.2 & 11.32 \\
\hline
\end{tabular}

given in Table 2 has been utilized to simulate two-layered structure.

\section{RESULTS}

When measuring the dielectric properties of a two-layer structure, the results are produced by the contribution of dielectric parameters in both layers. The measured sensing depth will be varied due to altering the aperture size of probe and thickness of upper layer. To demonstrate the impact of probe aperture size on sensing depth, we have been performed various simulations.

The results are illustrated in Fig. 2, 3 and 4 that relative permittivity and conductivity, respectively, have been obtained from the simulated S-parameter results of the probes with $2.2 \mathrm{~mm}, 0.9$ $\mathrm{mm}$ and $2.2 \mathrm{~mm}$ in aperture diameters. The dielectric properties have been retrieved for upper layer thicknesses from $0.1,0.2,0.3,0.5$ and $1.2 \mathrm{~mm}$ over the $500 \mathrm{MHz}$ to $2 \mathrm{GHz}$ bandwidth. In Fig. 2, the thickness of the upper layer skin was simulated at $0.4,0.6,0.8,1$ and $2 \mathrm{~mm}$ with a perfect electrical conductor placed underneath skin to evaluate the sensing depth of the probe. An additional upper layer skin simulation is added as a reference to the dielectric property of skin in which there no perfect electrical conductor is placed under the skin. The thickness of the reference upper layer skin is taken as $40 \mathrm{~mm}$ for all three probes. For the $2.2 \mathrm{~mm}$ probe, the results in show that, the dielectric properties of the upper skin layer decreases as the thickness increases. Furthermore, the upper layer skin thickness closest to the reference skin permittivity and conductivity is $2 \mathrm{~mm}$.

Similar simulation procedure is used for both $0.9 \mathrm{~mm}$ and $0.5 \mathrm{~mm}$ probes. Upper layer skin thickness of $0.2,0.4,0.6$ and $1 \mathrm{~mm}$ for the $0.9 \mathrm{~mm}$ probe and $0.1,0.5,0.7,0.9$, and $1 \mathrm{~mm}$ for the $0.5 \mathrm{~mm}$ probe were simulated. The results in Fig. 3 and Fig. 4 show that the dielectric properties of the upper skin layer decreases as the thickness increases for both the $0.9 \mathrm{~mm}$ and $0.5 \mathrm{~mm}$ probes.

In addition, the upper layer skin thickness closest to the reference skin permittivity and conductivity is $1 \mathrm{~mm}$ for the $0.9 \mathrm{~mm}$ and $0.5 \mathrm{~mm}$ probes respectively. To explain how the size of layer in close proximity has the dominant influence on measured dielectric properties, we plotted Fig. 5 that shows the relative permittivity and conductivity as a function of upper layer thickness for a 

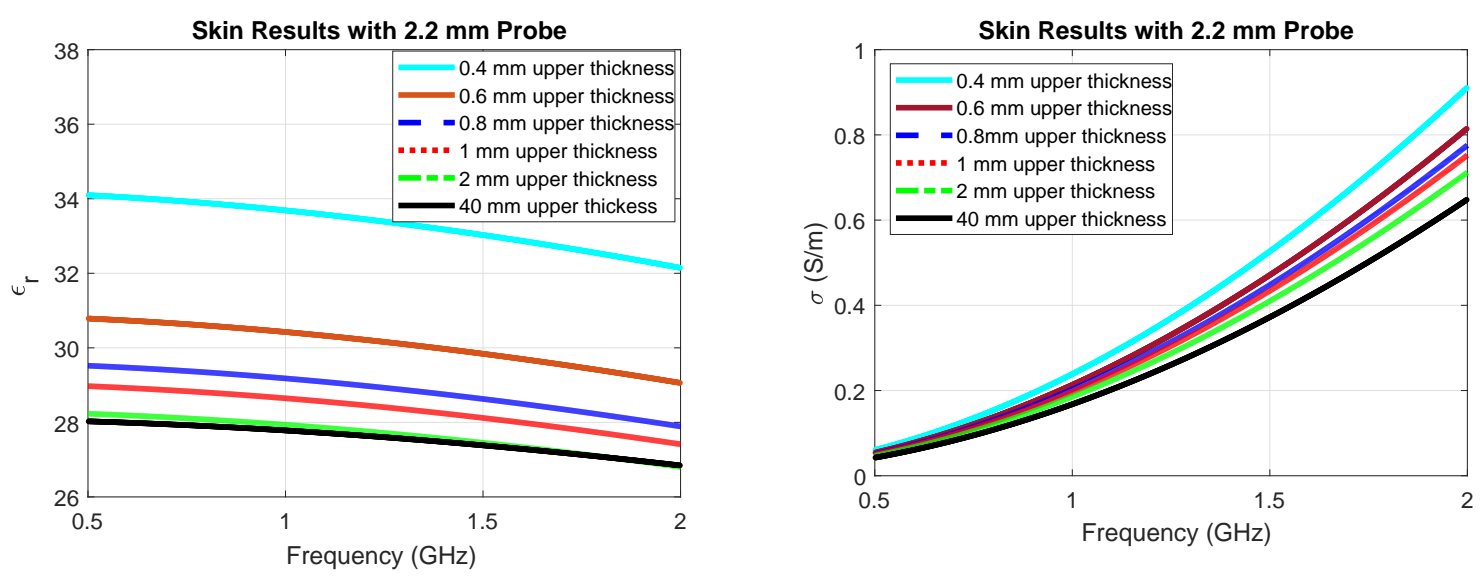

Figure 2: Obtained dielectric properties with $2.2 \mathrm{~mm}$ probe based on frequency for six different upper layer thicknesses: 0.4, 0.6, 0.8, 1, 2 and $40 \mathrm{~mm}$, respectively. (a) Relative permittivity and (b) conductivity.

Table 3: The thicknesses of skin tissue that can be achieved closest relative permittivity of two-layered structure to homogeneous skin structured

\begin{tabular}{cc}
\hline Aperture Diameter $(\mathrm{mm})$ & Thicknesses $(\mathrm{mm})$ \\
\hline 0.5 & 0.9 \\
0.9 & 1 \\
2.2 & 2 \\
\hline
\end{tabular}

representative frequency $500 \mathrm{MHz}$.Fig. 5 shows the plot of the upper layer skin thickness against the dielectric properties for the $2.2 \mathrm{~mm}, 0.5 \mathrm{~mm}$ and $0.5 \mathrm{~mm}$ probes at $500 \mathrm{MHz}$ frequency. Since the $2.2 \mathrm{~mm}$ probe has a larger aperture compared to the $0.9 \mathrm{~mm}$ and $0.5 \mathrm{~mm}$ probes, in general it is expected to have a higher sensing depth.
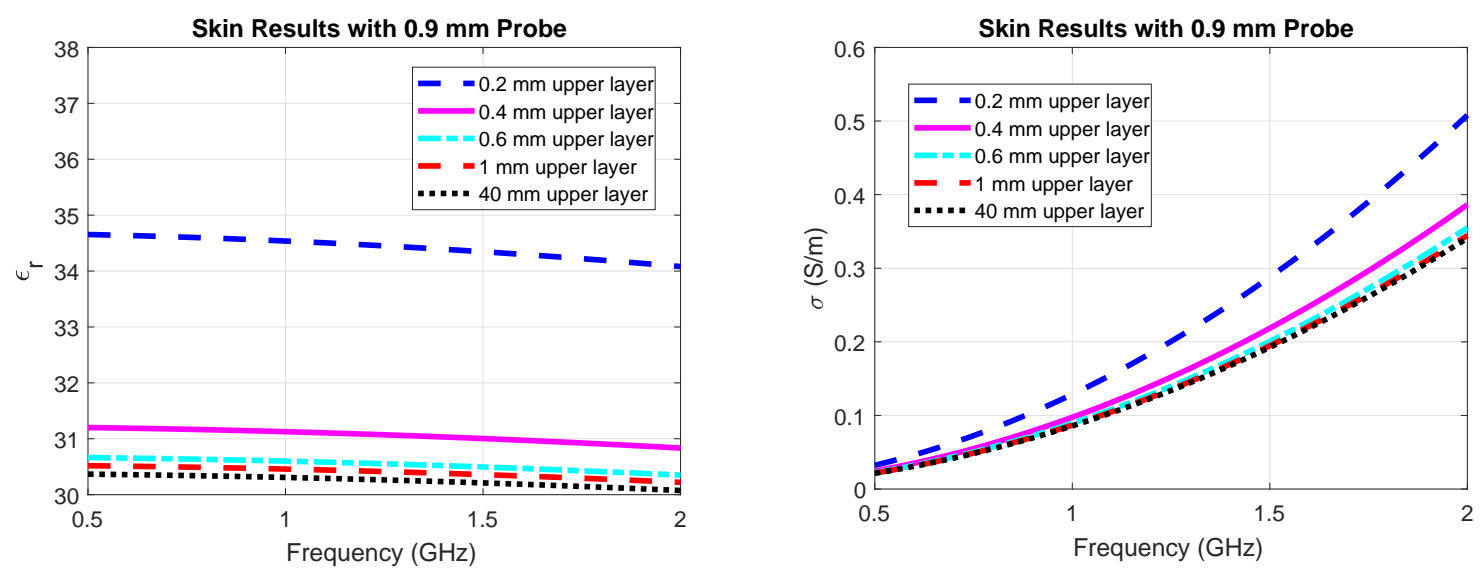

Figure 3: Obtained dielectric properties with $0.9 \mathrm{~mm}$ probe based on frequency for five different upper layer thicknesses: $0.2,0.4,0.6,1$ and $40 \mathrm{~mm}$, respectively. (a) Relative permittivity and (b) conductivity.

The results from the Fig. 5 shows that at $0.1 \mathrm{~mm}$ skin thickness, the $2.2 \mathrm{~mm}$ has a higher permittivity and conductivity than the $0.9 \mathrm{~mm}$ and $0.5 \mathrm{~mm}$ probes. Consequently, the $0.9 \mathrm{~mm}$ probe has a higher permittivity and conductivity than the $0.5 \mathrm{~mm}$. This results shows a good sensing depth agreement.

\section{CONCLUSION}

Biological tissues are composed of a layer structure, and in particular, the skin tissue is an example where it is difficult to detect dielectric parameters since each layer is very thin. Therefore, the knowledge of dielectric properties of a multilayered structure is vital to develop a new medical devices based on microwaves. In order to use for a variety of purposes that require a different 

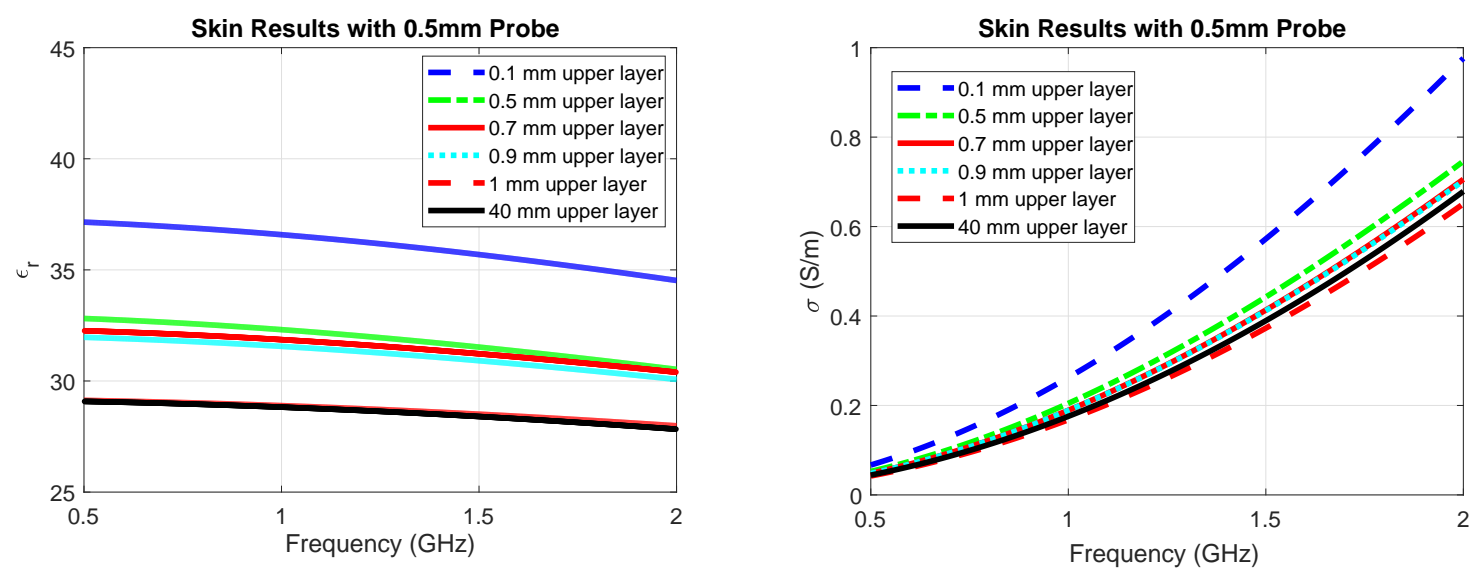

Figure 4: Obtained dielectric properties with $0.5 \mathrm{~mm}$ probe based on frequency for seven different upper layer thicknesses: $0.1,0.2,0.8,0.8,1,2$ and $40 \mathrm{~mm}$, respectively. (a) Relative permittivity and (b) conductivity.
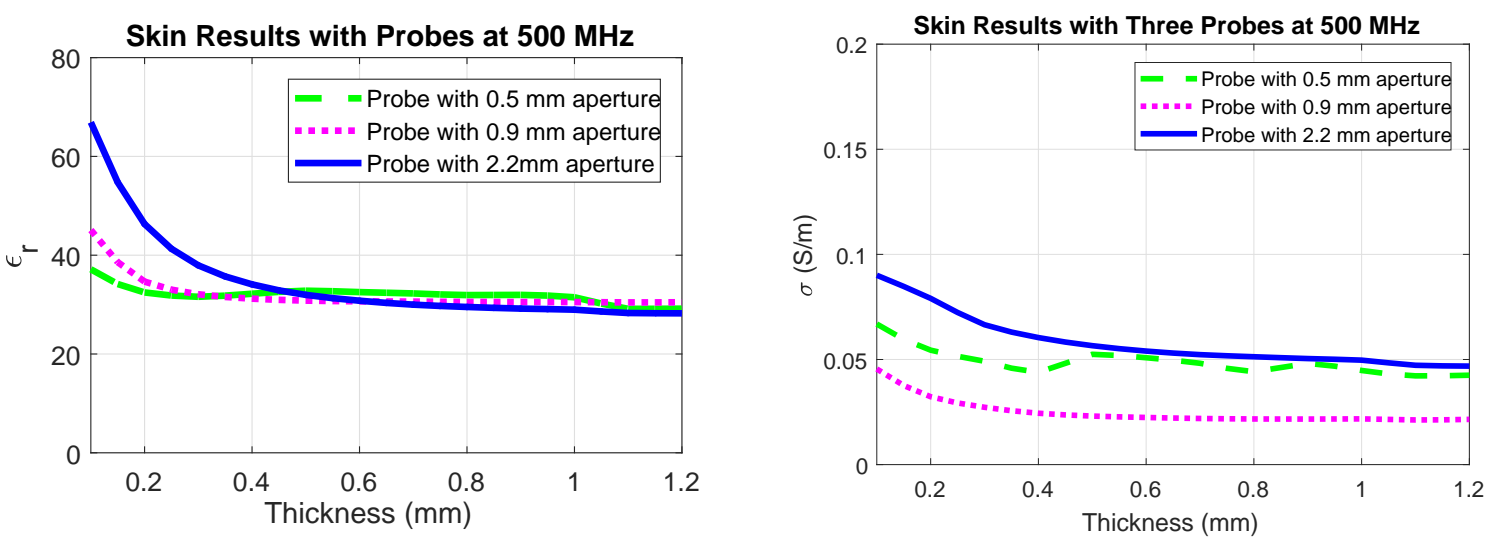

Figure 5: Obtained dielectric properties with 2.2, 0.9 and $0.5 \mathrm{~mm}$ probes based on upper layer thickness for two different frequency: $500 \mathrm{MHz}$. (a) Relative permittivity and (b) conductivity.

sensing depth, open-ended coaxial probes with different aperture sizes on two-layered structures has been designed by using CST Microwave Studio software. Obtained S-parameters has been run with in-house algorithm to acquire dielectric properties. In this paper, the main results of simulations of an open-ended coaxial probes with $0.5 \mathrm{~mm}, 0.9 \mathrm{~mm}$ and $2.2 \mathrm{~mm}$ aperture diameters on a two-layered structure has been demonstrated that the sensing depth can alter with probe aperture size. The results of the study can be utilized to analyze the effect of a coaxial probe dimension on the sensing depth.

\section{ACKNOWLEDGMENT}

This project has received funding from the European Union's Horizon 2020 research and innovation programme under the Marie Sklodowska-Curie grant agreement No 750346 and from the Istanbul Technical University grant 41554.

\section{REFERENCES}

1. England, T. S.,Sharples, N. A. A. "Dielectric Properties of the Human Body in the Microwave Region of the Spectrum," Nature, Vol. 163, No. 4143, 487488, 1949.

2. Cook, H. F., "The Dielectric Behaviour of Some Types of Human Tissues at Microwave Frequencies," Br. J. Appl. Phys., Vol. 2, No. 10, 295300, 1951.

3. Schwan, H. P., Li, K. "Capacity and Conductivity of Body Tissues at Ultrahigh Frequencies," Proc. IRE, Vol. 41, No. 12, 17351740, 1953.

4. Yilmaz, T., Kl, M. A., Erdoan, M., ayren, M., Tunaolu, D., Kurtolu, ., ... \& Cancan, G. "Machine learning aided diagnosis of hepatic malignancies through in vivo dielectric measurements with microwaves," Physics in Medicine \& Biology, Vol. 61, No. 13 5089, 2016. 
5. Popovic, D., McCartney, L., Beasley, C., Lazebnik, M., Okoniewski, M., Hagness, S. C, \& Booske, J. H., "Precision Open-Ended Coaxial Probes for in Vivo and Ex Vivo Dielectric Spectroscopy of Biological Tissues at Microwave Frequencies," IEEE Trans. Microw. Theory Tech., Vol. 53, No. 5 1713-1722, 2005.

6. La Gioia, A., Porter, E., Merunka, I., Shahzad, A., Salahuddin, S., Jones, M., \& OHalloran, M., "Open-ended coaxial probe technique for dielectric measurement of biological tissues: Challenges and common practices," Diagnostics, Vol. 8, No. 2, 40,2018.

7. Lazebnik, M., McCartney, L., Popovic, D., Watkins, C. B., Lindstrom, M. J., Harter, J., Sewall, S., Magliocco, A., Booske, J. H., Okoniewski, M.; et al., "A Large-Scale Study of the Ultrawideband Microwave Dielectric Properties of Normal Breast Tissue Obtained from Reduction Surgeries," Phys. Med. Biol., Vol. 52, No. 10, 26372656,2007.

8. Meaney, P. M., Gregory, A. P., Epstein, N. R., \& Paulsen, K. D. "Microwave openended coaxial dielectric probe: interpretation of the sensing volume re-visited," BMC medical physics, Vol. 14, No. 1, 3,2014.

9. Meaney, P. M., Gregory, A. P.,Seppl, J., \& Lahtinen, T. " Open-ended coaxial dielectric probe effective penetration depth determination," IEEE transactions on microwave theory and techniques, Vol. 64, No. 3, 915-923,2016.

10. Hagl, D. M., Popovic, D., Hagness, S. C., Booske, J. H., \& Okoniewski, M. "Sensing volume of open-ended coaxial probes for dielectric characterization of breast tissue at microwave frequencies," IEEE Transactions on Microwave Theory and Techniques, Vol. 51, No. 4, 1194$1206,2003$.

11. Porter, E., La Gioia, A., Santorelli, A., \& O'Halloran, M. "Modeling of the dielectric properties of biological tissues within the histology region," IIEEE Transactions on Dielectrics and Electrical Insulation, Vol. 24, No.5, 3290-3301,2017. 